\title{
Detecting vorticity in cohesive deformable granular material
}

\author{
Olivier Bouillanne ${ }^{1,2 *}$, Guilhem Mollon ${ }^{1}$, Aurélien Saulot ${ }^{1}$, Sylvie Descartes ${ }^{1}$, Nathalie Serres ${ }^{2}$, Karim Demmou ${ }^{2}$, and \\ Guillaume Chassaing ${ }^{2}$
}

${ }^{1}$ LaMCoS, Univ. Lyon, INSA-Lyon, CNRS UMR5259, F-69621, France

${ }^{2}$ Safran Aircraft Engines, Moissy-Cramayel, France

\begin{abstract}
Numerical models of granular materials are useful in tribology, and can be used to predict wear and friction in contacts. DEM-like simulations are used to model particles of third-body, which are partly wear debris from rubbing bodies. It has been shown that the third-body particles can have different flow regimes, depending on their mechanical properties. Among the different characteristics of flow regimes, agglomerate size seems to be crucial. A method based on vortex analysis used in fluid mechanics allows characterizing this cluster size. The results show that different vortex sizes can be observed during the simulation. In particular, it is observed that some vortexes of a characteristic size persist over time, and could be representative of agglomerates. These results pave the way for a better characterisation of the different flow regimes.
\end{abstract}

\section{Introduction}

\subsection{Tribology and third-body}

Tribology describes the interaction between two interacting surfaces in relative motion. This contact can lead to wear or fatigue, and then to the breakage of mechanical parts. For example, the contact between blades and disk in turbojet engines is critical, and full comprehension of wear in this situation is a challenge.

The third-body approach, developed by Godet [1], and then Berthier [2], is widely used in tribology. This approach considers that between the two rubbing bodies, a "third body" made of wear debris inevitably appears and modifies the interface frictional response. It accommodates velocities, transmits normal and tangential loads, and may protect or damage surfaces.

\subsection{DEM-like simulations for tribology}

Various granular models have been used to simulate third-body behaviours [3, 4]. These simulations showed that third-body has a considerable importance in the contact. Numerous parameters can influence its behaviour, and in turn, control wear of surfaces; such as thickness of third-body layer, cohesion and ductility of particles, etc.

The software MELODY developed in LaMCoS $[5,6]$ allows to simulate such complex granular material. In this software, grains can be rigid or highly deformable, with arbitrary shapes. Each particle is discretized, which makes it possible to use the laws of continuum mechanics, within the framework of large deformation hyperelasticity. For that purpose, we use a

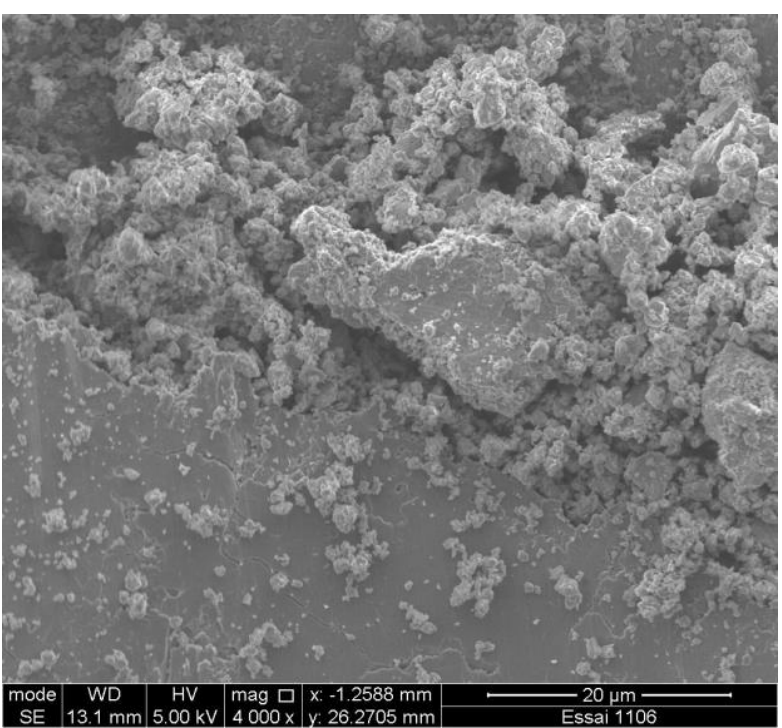

Fig. 1. SEM view of contact after friction test.

discretized weak form with meshfree shape functions. The code also uses a contact algorithm based on a piecewise linear description of the envelope of each grain in order to deal with arbitrary and ever-changing grain shapes.

This code was designed to model third-body in tribological contacts because SEM views of surfaces after friction showed that third-body has a granular aspect, as can been seen on Fig. 1. Depending on the stressed areas and friction parameters, it can also present different visual aspects: powdery, agglomerated, plastic, etc. Mollon [7] has shown that, by varying two parameters, deformability and cohesion, the flow of deformable particles simulated on MELODY can

\footnotetext{
* Corresponding author: olivier.bouillanne@insa-lyon.fr

A video is available at https://doi.org/10.48448/jebc-3z66
} 
accommodate shear following various kinematic patterns, which are consistent with these third body visual aspects.

Typical third-body simulations, carried out in an applicative case (blades-disk contact in turbojet engine), are shown in Fig 2. Two rigid or deformable bodies are placed at the bottom and the top of the simulated area. Between them, a collection of deformable and cohesive grains represents the third-body. A vertical pressure is applied on the upper body, while the lower body is fixed. When compaction is stabilized, a horizontal velocity is imposed to the upper body. Periodic boundaries are applied on the lateral sides. Three main flow behaviours are observed: plastic, granular (in which the grains deform very little and are not very cohesive), and agglomerated. In agglomerated flow, grains form cohesive clusters of various sizes with cooperative motion, as can be seen on Fig. 3. Agglomerates are hard to distinguish on a still image, but clearly appear on animated views.

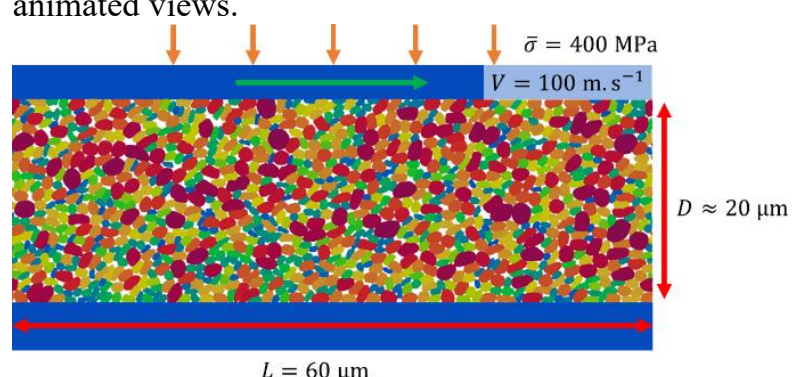

Fig 2. Diagram of third-body simulations made on MELODY.

Characterizing the size of the agglomerates would make it possible to analyse the consequences of the particle flow on the wear of the first bodies. To this end, the use of tools derived from fluid mechanics is documented here. They are based on the observation that the agglomerates tend to roll in the interface. The velocity fields hence show vorticity patterns. We present hereafter a methodology to characterize and quantify this vorticity.

\section{Methods}

\subsection{Vortex function identification algorithm}

In order to characterize these vortices, a vortex identification function is used. This function is inspired by mathematical descriptors which were proposed for similar purposes in fluid mechanics (see [8] and [9]). The function, called $\Gamma_{3}$, is a local value calculated on a velocity field. Let $P$ be a fixed point in the simulation domain. We define $\Gamma_{3}(x, y, R)$ at $P$ as:

$$
\Gamma_{3}(x, y, R)=\frac{\int_{M \in S}\left(\frac{\mathbf{V}-\mathbf{V}_{\text {ave }}}{\left\|\mathbf{V}-\mathbf{V}_{\text {ave }}\right\|}\right) \cdot \mathbf{V}_{\text {rad }} d S}{\int_{M \in S}\left\|\mathbf{V}_{\text {rad }}\right\| d S}
$$

Where $S$ is a disk of radius $R$ and of centre $P(x, y)$, $M$ is a point in this disk, $\mathbf{V}$ is the velocity of $\mathbf{M}, \mathbf{V}_{\text {ave }}$ is the average velocity in the disk, and $\mathbf{V}_{\text {rad }}$ is a velocity field corresponding to a rigid-body rotation of unit angular velocity and centred on $P$. Hence, $\Gamma_{3}$ can be seen

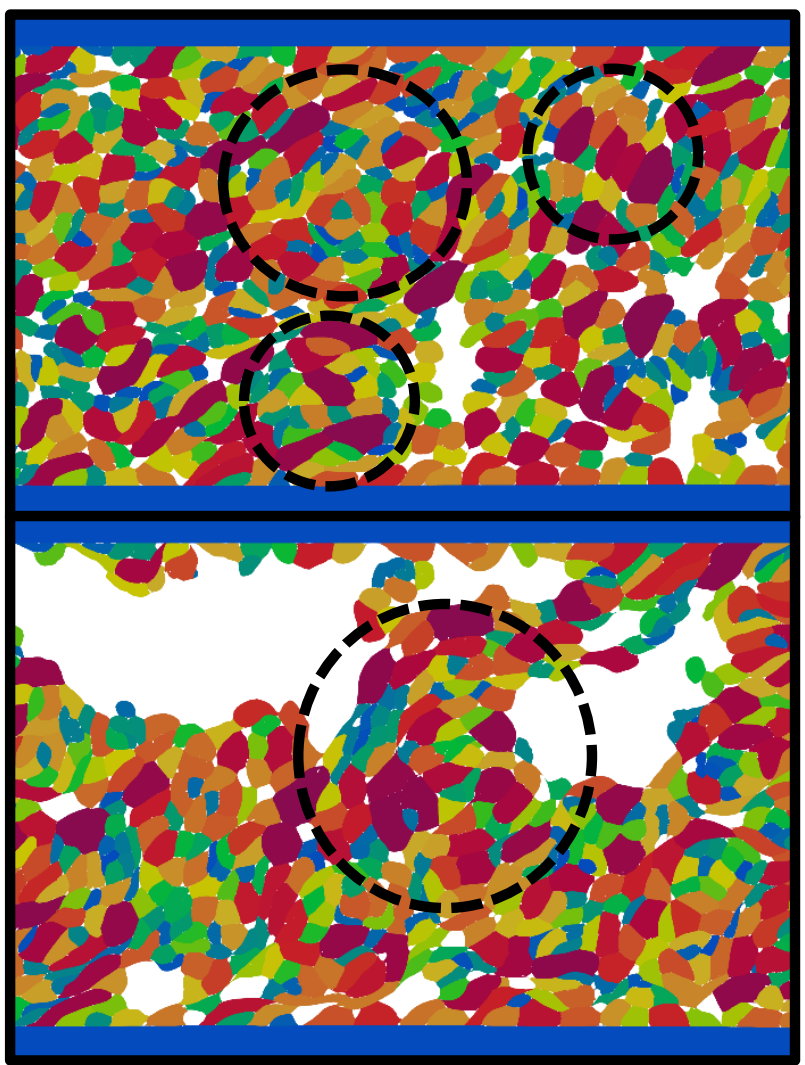

Fig. 3. View of two simulations, with formation of agglomerates. From top to bottom: small agglomerates (cohesion between particles of $2.2 \mathrm{GPa}$ ), and large agglomerates $(4 \mathrm{GPa})$. The Young's modulus is $1.6 \mathrm{GPa}$ for all simulations.

as the projection of the normalized de-trended velocity field in a certain circular region of the domain on a perfect rigid-body rotation of this region. By definition, $\Gamma_{3}$ is between -1 and 1 . A value of 1 means a perfect vortex that turns counter-clockwise and a value of -1 a perfect vortex that turns clockwise. $\Gamma_{3}$ measures rigidbody vortices, which differs from the vortices observable in fluid mechanics from which this method is inspired.

\subsection{Integration to granular simulations}

To apply this function to granular simulations, we first interpolate the velocity of the centre of each particle on a regular Eulerian grid, in order to obtain a regular representation of the velocity field in the third body at a given time. For a given value of $R, \Gamma_{3}$ is a function of the position $(x, y)$ of the point $P$, and can thus be mapped over the whole domain. We thus construct a 3D array of $\Gamma_{3}$ maps for different values of $R$, submit this array to Gaussian filtering, and perform a peak search to localize local extremums of $\Gamma_{3}$. These extremums are then considered as good approximations of vortices in the flow, with a certain position $(x, y)$ and a certain size $R$ (Fig. 4A-D). This operation is repeated for a large number of time-steps in order to build a database of vortices. Since $\Gamma_{3}$ represents the goodness of fit of a local velocity field to a rigid-body rotation, it will hereafter be called the "coherence" of this motion. 


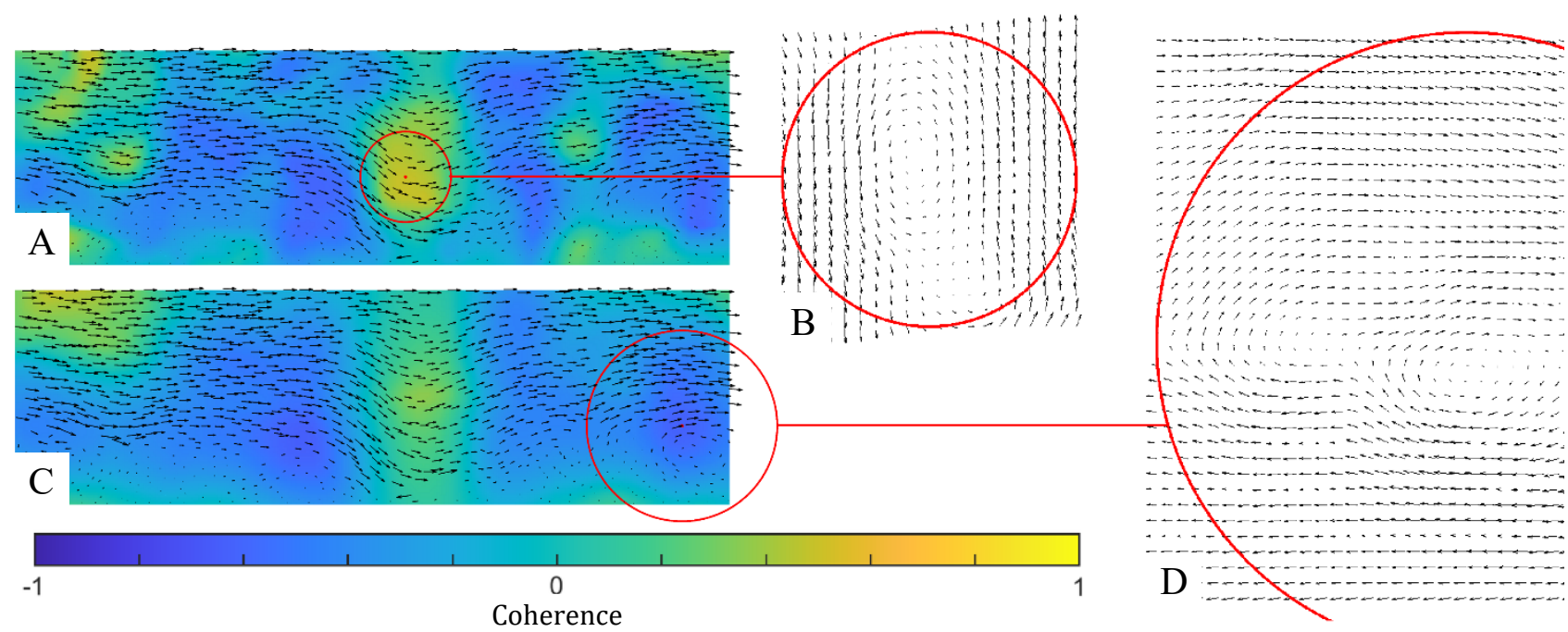

Fig. 4. A. Illustrative field of coherence for a radius $R=3.8 \mu \mathrm{m}$; B. Local maximum in this field, corresponding to a clockwise (i.e. retrograde) vortex; C. Illustrative field of coherence for a radius $R=8.0 \mu \mathrm{m}$; D. Local minimum in this field, corresponding to a counter-clockwise (i.e. prograde) vortex.

\section{Results}

Each detected event is characterized by its location $(x, y)$, by its radius $R$ and by its coherence $\Gamma_{3}$. As an additional property, we also compute its average angular velocity $\omega$, and normalize it by the steady-state angular velocity $\omega_{s S}$ corresponding to the large-scale shearing of the third body layer $\left(\omega_{s s}=V / h\right.$ where $V$ is the sliding velocity imposed on the upper first body and $h$ is the sample thickness). We thus obtain the normalized angular velocity $\widetilde{\omega}=\omega / \omega_{s s}$. If $\widetilde{\omega}=1$, the vortex rotates at the natural rate of shearing in the sample. $\widetilde{\omega}>$ 1 means that rotation is prograde and faster than shearing, $0<\widetilde{\omega}<1$ means that rotation is prograde and
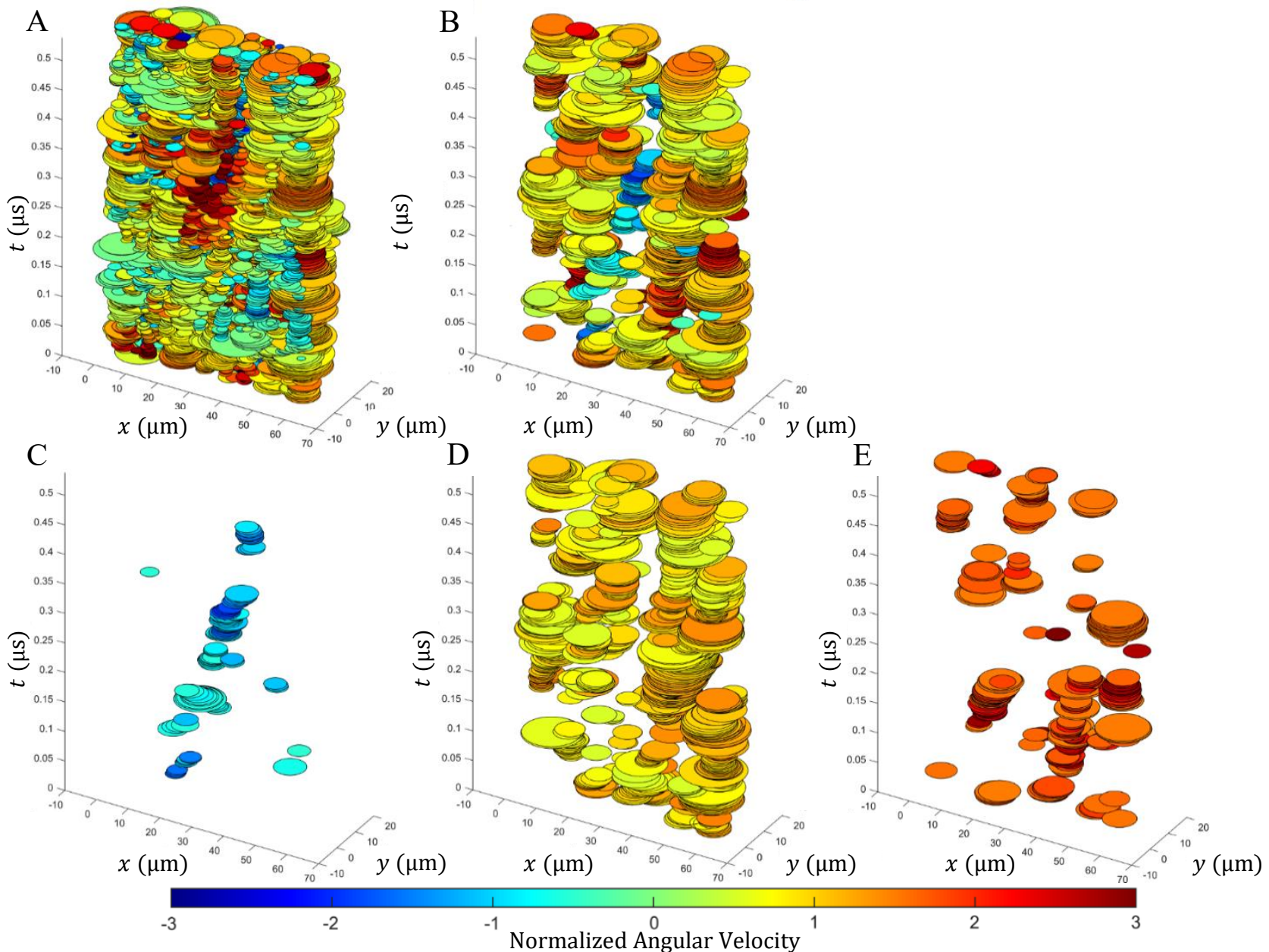

Fig. 5. A. Stacked representation of all detected vortices during a $0.5 \mu$ s period of shearing, represented as discs with the correct $(x, y, R)$ properties (time is on the vertical axis) and color-coded by normalized angular velocity; B. Vortices of A filtered based on coherence (larger than 0.4 or smaller than -0.4 ); C. Vortices of B filtered based on normalized angular velocity (lower than 0.5$)$; $\mathbf{D}$. Vortices of B filtered based on normalized angular velocity (between 5 and 1.5); E. Vortices of B filtered based on normalized angular velocity (larger than 1.5). 
slower than shearing, and $\widetilde{\omega}<0$ means that rotation is in the retrograde direction with respect to shearing. Numerical results are summarized in Fig. 5 for a short $(0.5 \mu \mathrm{s})$ period of a typical simulation where the granular sample has the following properties: cohesion of $2250 \mathrm{MPa}$ and deformability of $1590 \mathrm{MPa}$.

We observe that a large number of events are detected (Fig. 5A), but that many of them are of low coherence and may be ignored (Fig. 5B). Sorting events by angular velocity provides a tentative classification between three classes (Figs. 5C, D, and E). Vortices with $\widetilde{\omega}<0.5$ (Fig. 5C) are of limited number and of small size, but show a good consistency in terms of location and time, meaning that these events are rather persistent. Events with $0.5<\widetilde{\omega}<1.5$ (i.e. with a rotation rate close to that of the bulk shearing) are the most numerous, and have a larger average size (Fig. 5D). Finally, the events with $\widetilde{\omega}>1.5$ are usually smaller in radius, and nicely clustered in space and time, once again indicating a certain persistence during shearing.

Statistical data provided in Fig. 6A support this view. They indicate a general trend of increasing consistency of the vortices with their radii. A clear separation appears between a population of retrograde and prograde vortices, since almost no event is detected for $\widetilde{\omega} \approx 0$. It may either be because they do not exist or because the detection method is not able to catch them. The density map of Fig. 6B shows a main cluster of events in the region $\widetilde{\omega} \approx 1$ and $R \approx 3 \mu \mathrm{m}$, but two smaller clusters for $\widetilde{\omega} \approx 0.5$ and $R \approx 2.5 \mu \mathrm{m}$ on one hand, and $\widetilde{\omega} \approx 0.5$ and $R \approx 5-9 \mu \mathrm{m}$ on the other hand.

\section{Perspectives and conclusion}

These preliminary results only cover a short period of a single simulation, and cannot be yet considered as statistically consistent, but they provide a first
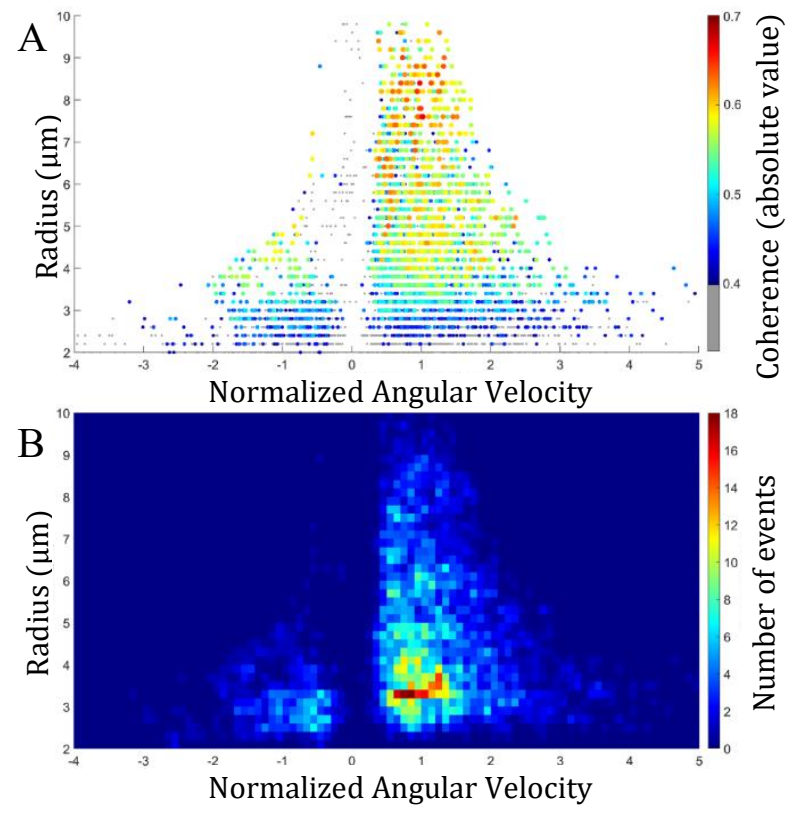

Fig. 6. A. All events sorted by radius R and normalized angular velocity, and color-coded by coherence; B. statistical distribution of events (restricted to coherence larger than 0.4 in absolute value). quantitative picture of the agglomeration and vorticity phenomena in sheared deformable and cohesive granular samples. The next step will be to process the database of detected vortices in order to retrieve a particular event from one-time step to the next, and thus to establish statistics on their life-duration. The question of a more consistent descriptor of the coherence of a given vortex than the one proposed here might also arise.

The influence of the temporal observation window is an interesting question. The structures we are trying to observe are structures that persist over time (agglomerates form and disappear on a large time scale during the simulation). Much shorter events, such as chains of forces or grain cycles, are not the object of this method of analysis. A study on the influence of the observation window must be done to validate the general approach.

Future extension of this methodology to other cases will make it possible to characterize the typical size of third-body agglomerates in various tribological situations, and to establish junctions with similar simulations performed at the atomistic scale, which allowed to derive a theoretical model for the typical length-scales of wear debris formations [10]. We expect to be able to relate such agglomeration patterns to the way third body damages and eventually wears the first bodies, by analysing the stress fields they generate in the surrounding mediums. The long-term purpose is to enrich existing wear laws by accounting for simple descriptors of the third body rheology.

The financial support of Safran Aircraft Engines is gratefully acknowledged.

\section{References}

[1] M. Godet, Wear 100, 437-452 (1984)

[2] Y. Berthier, L. Vincent, M. Godet, Wear 125, 2538 (1988)

[3] N. Fillot, I. Iordanoff, Y. Berthier, Tribol. Int. 40, 973-981 (2007)

[4] M. Renouf, H. Cao, V.H. Nhu, Tribol. Int. 44, 417-425 (2011)

[5] G. Mollon, Int. J. Numer. Methods Eng. 108, 1477-1497 (2016)

[6] G. Mollon, Comput. Part. Mech. 5, 517-527 (2018)

[7] G. Mollon, Tribol. Lett. 67, 120 (2019)

[8] L. Graftieaux, M. Michard, G. Nathalie, Meas. Sci. Technol. 12, 1422-1429 (2001)

[9] A. Berson, M. Michard, P. Blanc-Benon, Comptes Rendus - Mec. 337, 61-67 (2009)

[10] R. Aghababaei, D.H. Warner, J.-F.Molinari, Nat. Commun. 7, 11816 (2016) 